\title{
Personal Server Model for Real-Space Networking
}

\author{
WAKAYAMA Shirou ${ }^{1}$ KAWAKITA Yuusuke ${ }^{1}$ \\ KUNISHI Mitsunobu ${ }^{2}$ HADA Hisakazu ${ }^{3}$ MURAI Jun ${ }^{1}$ \\ 1 Graduate School of Media and Governance, Keio University \\ \{shirou, kwkt,jun\}@sfc.wide.ad.jp \\ ${ }^{2}$ Graduate School of Science and Technology, Keio University \\ kunishi@tokoro-lab.org \\ 3 Research Division, Digital Library Nara Institute of Science and Technology, JAPAN \\ hisaka-h@wide.ad.jp
}

\begin{abstract}
As the Internet population grows, not only computers but household electric appliances, such as VCRs and a refrigerators, will become connected to a network. The demand of connecting appliances to a network is increasing, however, other cheap objects are difficult because of the cost or technical problems.

In this research, we focus on connecting people to the Internet. We propose the Personal Server Model that sets up a personal agent on the Internet. An agent, we called "shell", obtains information by using sensors. Also, shell proves information via Internet. With this shell, any person can become an virtual Internet node. By treating person as an Internet node, applications which use the personal information can be developed easily like conventional Internet applications.
\end{abstract}

Keywords: Personal Server Model, Context Based Computing, Location Awareness, Real-Space Networking, RFID.

\section{Introduction}

The information appliance that has network connectivity is developed briskly. Not only electric appliances but also even homes and vehicles have connected to Internet[1]. Moreover, it is thought that there are many advantages of connecting other cheap and common object like coffee-cup to the Internet. The Real-Space Networking aims to connecting these object to the Internet and obtain information about these object via Internet.

The target object in this research has the following features.

- Object which has no Internet connectivity because of various reasons like cost, a size and so on.

- Mobile object.

- One object. In other words, nesting is not target.

Among the objects with such features, we especially focus on the "people". By connecting people to Internet, various kinds of information can be obtained. Moreover, a mighty change occurs in the computing environment.

However, there are numerous kinds of information that are personal information . For instance, schedules, contact addresses and context information such as walking, sitting, attending a meeting, driving a car and so on. The file in the one's home directory may be included. All personal information is important. It can become more convenient by using this information. Though, we will concentrate on the personal location information in this research.

Next, the two scenarios of the application using people's location information will be described.

Healthcare A patient does not appear in the hospital at a scheduled time for a medicine, and cannot contact. In this case, an ambulance can be dispatched to the place where the patient's is by grasping it's position. However, in the case with people, moving to places where cannot be grasped by GPS, such as underground and inside buildings, must also be considered. In addition, person can be use heterogeneous sensor to obtain various kinds of information. 
Alarm clock Even in a case where an alarm clock goes off, and the person is still in bed, the alarm clock can detect from the location information that the person is still sleeping. An alarm clock would then continue to ring until necessary.

\section{Related works}

The Active Badge[3] location system and The Active Bat system [4] are the first Location-aware Computing system. Active Badge uses IR receiver and Active Bat uses an ultrasound in order to detect location. In Active Badge and Active Bat, users and objects have cheap and small tags which receive IR or ultrasound in order to detect location.

Cricket Location Support System[5] uses ultrasound and cheap receivers in object in order to detect the location of object. Cricket use radio frequency signals additionally in order to time measurements. In Cricket, location is decided by triangulation relative to the beacons.

Recently, studies based on IEEE 802.11b has became major like robot location [6]. Since IEEE802.11b becomes an wireless network method which has wide usage, becomes cheap and small device. However if target object has no electronic power like cheap object, system can not detect its location.

Geographical Location Information (GLI)[7] is able to map a mobile entity on the Internet to a geographical location. The GLI system consists of home and area servers. These servers are managed based in a hierarchical server structure toward highly scalable system However, GLI system assumed absolute coordinate like GPS.

Auto-ID Center[8] propose 96bit Globally Unique ID within the very small chip. ID is read by radio frequency. ID has changed to IP address by using ONS which has hierarchical server structure like DNS, and ID sent to server shown by that IP address. By using this ID, Auto-ID can track object movement on transportation system.

\section{Personal Server Model}

This paper focuses especially on person. In this section, we describe our personal server model which handles personal information from the sensor at each own server. At first, limitation of system which become from environmental limitation and our consideration is shown. We then discuss two kinds of models in storing those personal information. According to these discussion, we describe about Personal Server Model.

\subsection{System Design}

Sensors are required in order to obtain information from real space. However, there are various kinds of device type of sensor like data set. In addition, the movement of person who are targeted in this paper becomes problem. As well, in order to discriminate person uniquely, it is necessary to have ID. We will describe about detail of assumption environment.

- Heterogeneous Sensors

Sensors are used for obtaining information around person, such as location information. In this research, we use not only the sensor which the user holds, but the sensor which is installed in the environment. In other words, according to user movement, the using sensor will be changed and can not be specified. Location information data sets are quite different by sensors. Therefore, this system treats location information as a simple label. However, the method of labeling is not a target.

- All person has Globally Unique ID(GUID) GUID is used in order to identify the person. However, we assume only uniqueness about GUID. However, structure of ID space and ID development is out of Target.

Model Discussion In these assumptions, we classify according to viewpoint of where to hold sensed information. There are two kinds of models.

1. Store information obtained from sensors at one centralized server.

2. Store information obtained from sensors at distributed server.

We describe below the reason why Distributed server is more sufficient. 
- Durability

If there is only one server, server down affects all members. If it is going to raise durability, it will be the cost of management and hardware. For example, it is necessary for high cost to monitor around-the-clock or make data base redundant.

- Cost

Concentration of bandwidth and storage is able to avoid. Moreover, each server can have cheap resources.

- Privacy Protection

Since information from sensor is personal, it is necessary to protect. Since processing distributes, it is hard to carry out management and surveillance. but distributed model has a problem.

- Target lost problem

Since server is distributed, sensor will lost where send information, and application also lost where from obtain information.

For the reasons mentioned above, we store information at distributed server. Since information from sensor could be personal, personal information should be stored in a personal server. Thereby, the personal server turns into the person's personal server. Furthermore, the server will become an agent of person on the Internet. Next, we describe the details of this model, Personal Server Model and SDP, which solve the "target lost problem".

\subsection{Detail information of Personal Server Model}

In this paper, we propose the Personal Server Model where each people have a Personal Server that stores personal information like Location Information. Since personals server are distributed, this Personal Server Model is reflected in discussion at section 3.1. Figure 1 shows this model.

Sensors sense each person's information, and send it to Personal Server. Personal Server accumulates the personal information. The other Internet nodes can access to the Personal Server in order to get the personal information. In other words, Personal Server will serve as proxy or agent in the person's virtual-space. We called this Personal Server as "shell" and, Personal Information in the shell as "ghost".

In Personal Server Model, we can say the shell is mapped object on Internet from person. According to that, when other Internet node obtains personal information, connect with other ordinary Internet manner like socket connection. Eventually, Internet Application, which use personal information can be developed easily.

Fig. 1. Personal Server Model

Fig. 2. System Component

\section{System Architecture}

In this section, we will describe the system architecture based on Personal Server Model. Figure 2 shows the system components which is half-tone dot meshing. We then describe about these system components.

\subsection{Sensors}

As mentioned, we assume using Heterogeneous Sensors in order to obtain user's personal information. We classify sensors into the following two kinds. 
- Self-directed type sensor

- Environmental installation type sensor

Self-directed type sensor is a sensor where the sensor and sensing target is same. For example, GPS sensor receives the electric wave from a satellite and obtains its position. Therefore, sensing target is GPS sensor itself. Although this type has high accuracy, in many cases, these devices tend to be quite expensive.

Environmental installation type sensor installed environment and sense the target when the target enter within the limits of sensing. For instance, there are many studies on using radio frequency (RF). Since these sensor detect cheap target devices by using RF, they can obtain location information. While sensor itself becomes expensive, target devices are cheap. Consequently, this kind of sensor is useful to operate as infrastructure. In addition, it must be noted that, when we use this kind of sensor as a location sensor, obtained information becomes relative location. For example, GPS uses latitude and longitude and altitude. However, RF sensor cannot get these absolute coordinate, but only labels such as room name.

As mentioned above, this research assumed on the Heterogeneous Sensor environment. Hence, it is necessary to accommodate to two kinds of sensors. These sensors have different data sets. However, the difference in the data-set are solved by location-modeling, labeling like "LOBBY" or using R2 system[14].

4.2 shell

Shell is the Internet node that hold ghost as mentioned above. The other Internet node can obtain personal information such as location information of the owner of that shell by accessing to the shell.

Internet nodes which wants to obtain ghost sends a request message to the shell which has the ID of the target person. Request message must contain the ID of the person whom is using that Internet node. Shell returns ghost described by PML to the request node. Basically, shell has this function. In subsection 4.4, SDP which provide IP address from ID will be described.

It may be setup the shell anywhere on the Internet. Moreover, the shell always needs to be connected to the Internet. However, as long as it is a short time, the shell may move. It becomes impossible to obtain the person's information in the meantime.

Privacy Protection Personal Server may be set up anywhere on Internet. Many Internet nodes connect to the shell. Since shell has the personal information like location information, privacy protection is required. Moreover, only one Personal Server has managed his personal information. Since the personal information managed unitary on one Personal Server, when performing privacy protection, it is necessary to protect only one Personal Server.

We prepare an access control mechanism, in order to realize privacy protection. When requesting personal information, internet node must include GUID in a request message. The access control mechanism inside the shell changes sending personal information to according to GUID contained in a request message. Shell may return nothing if request is invalid. Thereby, information cannot be passed to a request from an unexpected partner or the partner who does not want to give.

\section{3 ghost}

Ghost is Personal Information created from sensors. We propose Person Markup Language(PML) in order to describe the ghost. Ghost itself is just an abstract word about personal information. When shell exchange personal information with other Internet node, PML is used.

PML PML is XML[9] based language for describing personal information. PML must contain a core part. A core part contains GUID, Location Infomation, and Timestamp that obtained the last Location Information.

Figure 3 shows an example of a simple description of one person.

The core section of PML example in Fig.3 shows also that this person has ID HZMHMGL and that his actual location is in the room called t20, and the Timestamp from which the Location was last obtained.

Moreover, PML can describe various kinds of information in addition to the core section. Figure 3 shows also person name and e-mail address. Thus, PML is extensible so that various kinds of information may be expressed. Ghost described by PML is updated on the fly by the sensors or user himself. 


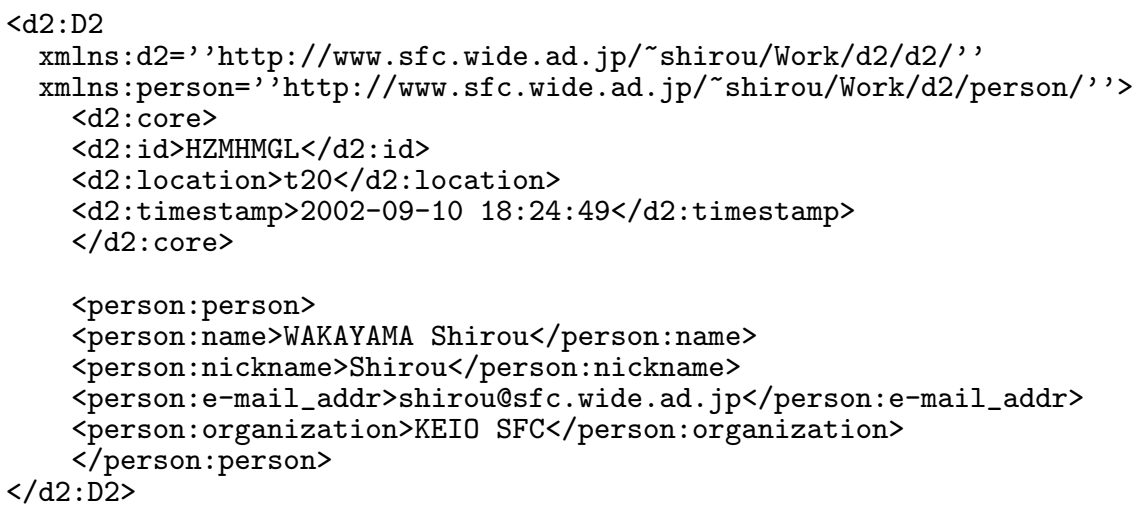

Fig. 3. an example of ghost described by PML

\subsection{Shell Discovery Protocol(SDP)}

Other Internet nodes and sensor nodes need to know a shell IP address, in order to access to a certain person's shell. In this paper, as mentioned above, it is assumed on all persons holding Globally Unique ID (GUID).

Shell Discovery Protocol (SDP) changes person's GUID into that person's shell IP address. We can say that SDP changes GUID that is independent of location into IP address depending on a location.

Group of nodes using SDP handles Shell Discovery Protocol. These SDP groups are overlay network on Internet. There connect each other by P2P network like CAN[10], and they exchange the conversion table to shell IP address from ID. Node that has participated in SDP Group can convert the ID to that shell IP address. Since SDP consists of P2P overlay network, a little resource is required for each node in SDP group.

However, it is not desirable on privacy protection that other unnecessary nodes hold the conversion table to IP address from ID. Therefore, Conversion table has no ID itself but one-way hashed ID such as MD5. Only node which knows right ID can map certain IP address. Thereby, even if shell IP address understands the middle node of SDP group, it can not know whose ID it is. In addition, even if it connect the shell IP address, it will be flipped in the Access Control section of shell.

Beyond that, not only Internet nodes, but a sensor node also uses SDP. A sensor node may have restriction in the memory capacity, calculation capability, network bandwidth by using wireless, and so on. Therefore, the conversion table from Hashed ID to IP address is also transferable to other SDP node.

\section{Implementation}

In this section, we will describe at Implementation. This system is consists a sensor part, shell, and an application part. A sensor part transmits the information on Real-Space to the shell. A shell holds the received information as ghost in DB. Moreover, it has GUID and an IP address in shell. In this implementation, in order to realize many shells, IPv6 was used.

Next, we then describe about each part of system component detail.

\subsection{Sensors}

We used two kinds of devices as Location Sensor. One is RF-Reader and the other is LIN6-MA. RF-Reader is sensor on real space, and LIN6-MA can provide location information from network prefix.

RF-Reader We use the spider tag of RF-Code[15] which is a system for detecting a personal location.

The cost of sensor construction is one of reasons for having chosen RF-Code. Although other systems, such as Active Bat, have very high accuracy. however, huge cost is required. We think 
s personal Location should be known about per room. Therefore, cost becomes important rather than accuracy.

Therefore, the Self-directed type sensor which needs a high cost device for an individual user is unsuitable. We selected RF-Code as an Environmental installation type sensor, and all users always carry a cheap and small tag. The tag is shown in Figure 4.

This RF-ID tag is a tag carrying its own battery, each with a Globally Unique ID, and sending the ID by using $300 \mathrm{MHz}$ radio frequency. Tag sends ID once every 3 seconds, and when RF-Base Station catches this ID, it detects that a user is in detection within a sensing area of that RF-Base Station . Figure 5 shows RF-Base Station.

Furthermore, we prepared three kinds of the antenna attached to RF-Base Station. Since this sensitivity different, the antenna was properly used according to the size of the each room. Thereby, one RF-Base Station area can cover mostly equivalent to one room. Moreover, the large room installed two or more leaders.

RF-Reader set consists of an Mobile Gear[11] for connecting network and RF-Base Station. Mobile Gear connects to network by using Wireless LAN; added to this, RF-Base Station and Mobile Gear have a battery. RF-Reader set is completely movable by wire free.

Mobile Gear connect to RF-Base Station by using RS-232C, and sends GUID sensed by RFBase Station via network to shell of owner known by ID. Mobile Gear sends the label of RF-Reader set additionally. According to this label, location information is determined.

Moreover, the Mobile Gear is operated by NetBSD/hpcmips. Reliability is increasing by using not WindowsCE but NetBSD. In addition, it is manageable from remote host.

LIN6-MA In LIN6[12], unique ID of a meaning called LIN6ID is assigned to each host. After a host can assign network prefix, LIN6ID and network prefix will be compounded and an IPv6 address will be generated automatically. By registering this IPv6 address into Mapping Agent (MA), a host notifies a communication partner of in which network it exists, and realizes Location Independency.

If a user connects Note PC with the network, LIN6ID and network prefix will be compounded and an IPv6 address will be generated automatically. This IPv6 address is sent to MA with LIN6ID. MA, which received the IPv6 address transmits an IPv6 address and LIN6ID to the process called NLX (Network Location eXchanger). NLX can know owner of the note PC by LIN6ID, and the network by network prefix. As shown below in subsection5.3, the network that has different prefix for every room in this experiment, it is possible to get to know the location of Note PC by network prefix.

\subsection{User Interface}

Two kinds of interfaces were prepared as an interface to shell. One is interface for human, the other is used to develop.

Web Interface We prepared the web interface to connecting shell and obtaining location information. Users can get the following things through this web interface. 
- Search Location Information

By specifying a location, it is possible to get the List of users who are near that location. From this list, the situation of that location and how many participants are gathering can also be known via network.

- Search Information from Location

By specifying a location, you can get list of users who exist near that location. From this list, the situation of that location and how many users are gathering can be known also via network.

- Tracking Note-PC

We are able to find out the location where Note PC is put based on LIN6ID which described below.

- Acquisition of Shell IP address

It is possible to perform SDP(Shell Discovery Protocol) through a web interface. Thereby this interface, it is also possible to get shell IP address and to obtain information from shell directly.

Library Moreover, we prepared library of the C language for accessing shell so that it might be easy to develop the application which uses location information. This library contains the functions, which can get the IPv6 address of shell using SDP, and connect to shell and acquires PML. In addition, SDP command line program was also created.

\subsection{Experimentation}

The experimentation using this system was conducted during the WIDE-camp in September 2002. WIDE-camp is research conference held at a Hotel where member of WIDE Project[13] gather and open BoFs to have discussions. 274 people participated in this experiment during 3 nights and 4 days.

Figure 6 shows the Map. Each room are labeled with a name like "BOF1". Location information of Participants is expressed using this labels. In this experiment, labeling was performed manually. The white circle in figure 6 shows the place in which RF-Reader where setup as mentioned below. Note that figure 6 will be used at section 6 .

Fig. 6. Hotel Map

For the Location detection by LIN6 mentioned above, segments is divided for each and every room to show a prefix for each room. Furthermore, in this experiment, since the host for many shells was not able to be prepared, one host was attached two or more IPv6 addresses as Alias. Then, shells for all the members was prepared virtually.

Sensing Devices As described above, we use RF-ID as Location sensor. When two RF-Reader sets and people enter between them, it becomes a most important problem when choosing its location.

Moreover, Hotel used at WIDE-camp had thin walls, and radio waves passed through them easily. Therefore, a RF-reader set will receive an ID ranging over the room. Consequently, people may seem to have moved even though they didn't.

However, many BoFs and presentation are made at WIDE camp. In this circumstances, we can assume that once people goes into the room, they will not leave that place for a certain period of time. 
Therefore, the position judgment algorithm in consideration of hysteresis was adopted. This algorithm can reduce miss detection of movement.

In this experimentation, we used LIN6 as another location sensor.

In this experiment, to support when a participant has two or more PCs, every 16 LIN6ID was assigned every participant.

\section{Evaluation}

In this section, we will describe about Evaluation of this system. First, we will start about accuracy of sensor. The three kinds of evaluation will be shown that are Human Location Tracking, Human behavior and General Movement. Next, we will discuss about Personal Server Model.

\subsection{Human Location Tracking}

As quantitative evaluation, we compared tracked one person movement and actual movement. Figure 7 shows sensed location and actual location while 24 hours (actually, 18 hours). In this figure, room name was changed to room number.

Fig. 7. Sesnsed Movement and Actural Movement

Although we can say that this figure showed person's general place, there are still many problems. However, the problem of such accuracy originates in the sensor itself, and will be solved by improvement in sensor technology.

\subsection{Human Behavior Observation}

Fig. 8. Number of People of room BOF2

Fig. 9. Number of People of room LOBBY3

We select at two rooms it show the characteristic motion of people and these graphs is shown in Figure 8 and 9. These figures which mean how many people are in the room for every time. As for Figure 8, BOF2, people are beginning to be detected from the 11:30 time by which BOF was 
started. Moreover, it is not detected at all midnight. However, in Lobby3 which is a passage, it is always detected except for early morning. A motion of an overall person can be grasped from these figures.

General Movement Matrix of table 1 shows movement of people about "from where" and "to where". This table shows whether Location-Modeling could be performed by combining the map of figure 6 . The still more efficient location detection technique can be used now like this experiment not by the short period of time but by applying in the long term.

Table 1. Movement Table

\begin{tabular}{|c|l|l|l|l|l|l|l|l|l|l|l|l|}
\hline From To & BOF1 & BOF2 & BOF3 & PLENARY1 & PLENARY2 & LOBBY1 & LOBBY2 & LOBBY3 & LOBBY4 & NOC & FRONT & Total \\
\hline BOF1 & - & 24 & 0 & 14 & 20 & 11 & 28 & 54 & 0 & 18 & 6 & 175 \\
\hline BOF2 & 18 & - & 12 & 24 & 28 & 24 & 37 & 51 & 0 & 13 & 6 & 213 \\
\hline BOF3 & 1 & 13 & - & 1 & 1 & 1 & 5 & 9 & 0 & 0 & 7 & 38 \\
\hline PLENARY1 & 36 & 32 & 6 & - & 188 & 83 & 134 & 173 & 4 & 43 & 21 & 720 \\
\hline PLENARY2 & 20 & 29 & 2 & 160 & - & 47 & 115 & 157 & 2 & 43 & 23 & 598 \\
\hline LOBBY1 & 11 & 28 & 1 & 46 & 61 & - & 129 & 61 & 0 & 26 & 10 & 373 \\
\hline LOBBY2 & 23 & 39 & 4 & 76 & 117 & 133 & - & 102 & 2 & 34 & 14 & 544 \\
\hline LOBBY3 & 55 & 65 & 11 & 78 & 153 & 59 & 108 & - & 5 & 113 & 47 & 694 \\
\hline LOBBY4 & 1 & 0 & 0 & 0 & 5 & 3 & 1 & 3 & - & 0 & 1 & 14 \\
\hline NOC & 18 & 19 & 0 & 22 & 41 & 24 & 33 & 104 & 0 & - & 5 & 266 \\
\hline FRONT & 9 & 5 & 2 & 10 & 25 & 9 & 13 & 46 & 0 & 5 & - & 126 \\
\hline
\end{tabular}

\subsection{Model Discussion}

This research aim at small, mobile object which is not Internet node like people to connect Internet. In order to that, we propose Personal Server Model.

Personal Server Model propose to store personal information in distributed personal servers, named shell. There are points to fill described in section3.1.

- Durability

Still one shell down, the other shell has no effect. This durability is important to minimize the damage and reduce maintaining cost.

- Cost

Little storage and bandwidth are required per one shell. However, shell should connect to Internet all the time.

- Privacy Protection

Since shell has Access Control part, Privacy Protection is maintaining. Certainly, the user has to pay the cost of managing Access Control List. However, we think it is reasonable.

- Target lost problem

Shell Discovery Protocol and GUID can resolve this problem. Additionally, since SDP group has only hashed ID, Privacy Protection is satisfied.

Then, we will discuss whether Personal Server Model achieves this purpose.

- Connecting person to Internet?.

Shell that similar to virtual agent on Internet can acquire personal information on real space from sensors. By using shell, access was made possible similarly to the conventional technique of socket connection or telnet.

- Is the personal information in real space offered?.

We proposed PML, which can treat and exchange personal information easily.

According to these discussions, Personal Server Model is suited to realize connecting person to Internet. Shell, PML and SDP that compose Personal Server Model of assistance to person.

\section{Conclusion}

This research aims being connecting the person whom it did not connecting network to Internet.

We proposed Personal Server Model which uses an agent on the Internet as a communication node, named "shell". Sensors sense personal information like location, and send to his shell. Then 
shell holds this personal information. If other Internet node wants to obtain personal information, connect to the shell, and can get personal information.

According to this Personal Server Model, durability, low cost and privacy protection were performed. In addition, internet applications which use the personal information on Real-Space by the same technique as usual internet application can be developed.

\subsection{Experimentation}

In order to establish Personal Server Model, the actual proof experiment was conducted. In this experiment, RF-ID was used for Location-Sensing, 274 participants' movement was detected, and it held to each shell.

Moreover, web Interface for using this location information and library for using from the $\mathrm{C}$ language were prepared. Thereby, application which uses personal location information can be developed easily.

\subsection{Future Work}

Present shell has only a function, which is Access Control and holding ghost described by PML. However, shell holds various kinds of personal information. Therefore, more convenient environment will be able to be offered if it becomes possible to extend the function of shell easily if needed.

In addition, we use two kinds of sensor devices in this implementation. We cannot say heterogeneous sensor environment by using only two kinds of devices. Since that, we will develop more sensor devices and more applications.

Although especially Personal Server Model has focused to people, it is applicable also to the simple device which does not have Internet connection nature by the reasons of cost and so on. Therefore, it is necessary to extend the target range of this research as a Real-Space network will spread from now on. It will be necessary to specifically perform further extension of PML.

\section{References}

1. K.Uehara, Y.Watanabe, H.Sunahara, O.Nakamura, J.Murai, "InternetCAR -Internet Connected Automobiles-", Proc. of INET'98, Jul 1998

2. B. Schilit and N. Adams. 'Context-aware computing applications In Proceedings Workshop on Mobile Computing Systems and Applications. IEEE, December 1994.

3. R.Want, A.Hopper, V.Falco,and J.Gibbons. The Active Badge location system. ACM Transactions on Information Systems, 10:91-102,Jan.1992

4. Mike Addlesee, Rupert Curwen, Steve Hodges, Joe Newman, Pete Steggles, Andy Ward, Andy Hopper. Implementing a Sentient Computing System. IEEE Computer Magazine, Vol.34, No. 8, August 2001, pp. 50-56.

5. N. Priyantha, A.Miu, H. Balakrishman, and S.Teller. The Cricket compass fo context-aware mobile applications. In Proc. of the 7th Annual ACM/IEEE International Conference on Mobile Computing and Networking(MOBICOM 2000),pages 1-14, Rome,Italy,July 2001.

6. Andrew M. Ladd,Kostas E. Bekris,Algis Rudys,Guillaume Marceau,Lydia E. Kavraki and Dan S. Wallach,"Robotics-Based Location Sensing using Wireless Ethernet" In Proc. of the 7th Annual ACM/IEEE International Conference on Mobile Computing and Networking(MOBICOM 2002).

7. Yasuhito Watanabe, Atsushi Shionozaki, Fumio Teraoka, Jun Murai, "The design and implementation of the geographical location information system.", Proc. of INET'96. Internet Society, June 1996.

8. Kevin Ashton et al: http://www.autoidcenter.edu

9. XML: eXtensible Markup Language http://www.w3c.org.

10. Sylvia Ratnasamy, Paul Francis, Mark Handley, Richard Karp, Scott Shenker, A Scalable ContentAddressable Network

11. Mobile Gear MC/R550 NEC Corporation. http://www.nec.co.jp/press/en/9803/1101.html

12. Mitsunobu Kunishi, Masahiro Ishiyama, Keisuke Uehara, Hiroaki Esaki, Fumio Teraoka, LIN6: A New Approach to Mobility Support in IPv6, International Symposium on Wireless Personal Multimedia Communication, 2000

13. WIDE Project http://www.wide.ad.jp

14. Shirou WAKAYAMA, Takeshi IWAMOTO, Nobuhiko NISHIO, Hideyuki TOKUDA, Design and Implementation of Supporting Software for Sensor-Device Independence,DiCoMo,2001. Japanese Paper.

15. RF-Code http://www.rf-code.com 